\title{
Online monitoring of radiation damage in optical fibers
}

D. Bisello(1), P. Camarri( ${ }^{(2)}$, M. Cobal(2), H. Grassmann ${ }^{(1)}$, S. Leone ${ }^{(2)}$

(1) University and INFN Padova, V. Marzolo 8 Padova, Italy

(2) University and INFN Pisa, V.Vecchia Livornese 582/a S.Piero a Grado (Pisa), Italy

Presented by Marina Cobal

We describe a setup which is able to monitor the behavior of optical materials during irradiation. We present measurements of the change in transmission for optical fibers and the recovering after irradiation. A measurement precision of about $2 \%$ is obtained.

\section{INTRODUCTION}

In order to measure the effects of radiation on materials during the irradiation process we designed a setup which has, as main goals :

- small systematic errors, also over a long period of time.

- capability to measure different parameters simultaneously, like for example the transmission of fibers at different wavelengths of light.

- modular system with potential to upgrade, in such a way that a large number of different items can be studied at the same time.

- compactness and easy handling.

A device with those properties can provide detailed knowledge useful for the study and understanding of the fundamental processes caused by radiation. It can also be used to do a systematic empirical search for the best available material.

We studied the relative transmission of several plastic lightguiding fibers at different wavelenghts. However the setup could, without too many modifications, also be used to measure transmission or light emission of any other kind of scintillator or lightguide.

A first engineering run with a simpler version of the setup was described in ref.1.

\section{BASIC DESIGN AND EXPERIMENTAL SETUP}

Light of a certain wavelenght is transmitted to a photodiode through an optical fiber. The fiber is partially exposed to a radiation field. The current produced in the photodiode is then amplified and measured.

Radiation might damage the light emitters, the photodiodes or the preamplifiers. The electronics by itself could be unstable. Therefore an important part of the setup is the monitor system. To monitor changes in the output signal which are not due to a change in the transmission of the fiber, we used photodiodes viewing the light emitters directly, without any fibers in between. In addition electronic testpulses of constant amplitude are sent to the input of an amplifier in order to monitor changes of its amplification separately. The whole setup is controlled by an IBM personal computer via $\mathrm{Ca}$ mac.

The input light signals were provided by LED's emitting light pulses and by a contin- 


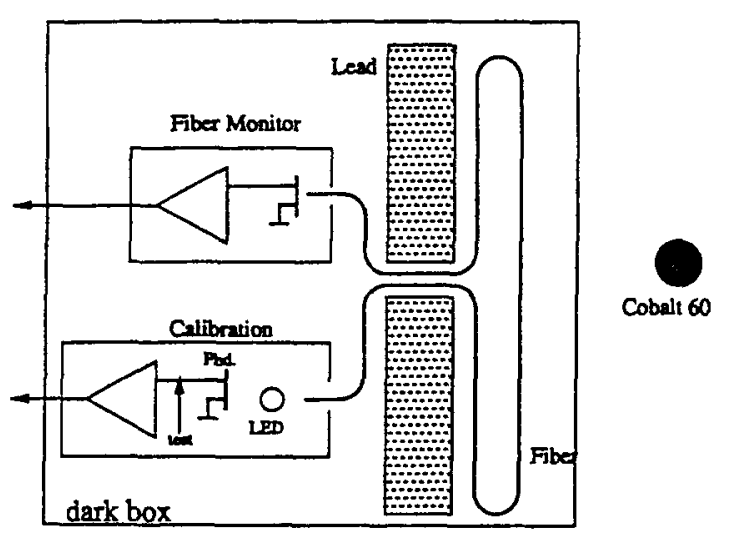

Figure 1: Schematic view of the experimental setup.

uously radiating lamp. For reading the output light signals, amplifiers in DC and in AC mode have been used. AC amplifiers could be potentially more precise, they can operate even in the presence of day light for example. DC amplifiers are easier to build and cheaper.

In our experimental setup the input light pulses were provided by red and green LED's (General Intrument green MV5453 and red MV5753, maximum of transmission at $582 \mathrm{~nm}$ and $635 \mathrm{~nm}$ respectively) and by a 4.5 volt lamp. The input signals provided by the pulsed LED's were read by photodiodes ( Hamamatzu Si790 ) equipped with $\mathrm{AC}$ amplifiers ( PHI OP27 in the first stage, National Semiconductor LF357 in the second stage ) The LED's were also directly viewed by a photodiode, as well the continuous light from the lamp.

Figure 1 shows a schematic drawing of the device. The light sources as well as the photodiodes and the amplifiers are behind a lead shielding consisting of about $10 \mathrm{~cm}$ of lead bricks. The fibers are traversing the lead shielding and are exposed to the radiation over a length of about 1 meter.

The measurements are done in air.

\section{THE SOURCE}

As a radiation source we used a 5400 Curie ${ }^{00} \mathrm{Co}$ source at the FRAE-CNR laboratory of Legnaro (Italy). The source consists of twenty radioactive slugs assembled and sealed in a cylindrical capsule with diameter 1.25 inches, and lenght 2 inches. This capsule is inside a lead container and can be brought out of the container by remote control. The irradiation facility is a panoramic irradiator, which emits a radiation beam 2 inches deep at the cylindrical surface of the source, through a 360 degree horizontal arc, and confined to a 257 degree spherical zone above the top surface of the sourcehead ( see ref.2) The facility is allocated in a shielded room of about $3 \mathrm{~m}^{*} 5 \mathrm{~m}$ in size, in which we put our setup.

\section{MEASUREMENT PROCEDURE}

The exposure to the source was done in two steps. For the first 16.1 hours of irradiation the distance between fiber and source was $43 \mathrm{~cm}$. Then we decreased the distance to $26 \mathrm{~cm}$ in order to increase the intensity of the radiation. After another 22.5 hours, at $t=39$ hours, we had the source switched off for 48 minutes, switched on for 16 minutes and then had it off for the rest of the experiment. This short reactivation of the source was done for making various checks and for making some precision measurement on a very short time scale (discussed in figure 6).

After the source having been switched off the fibers were observed for an additional 28.1 hours in order to study recovery effects.

\section{RADIATION LEVELS}

The alternated radiation intensity behind the absorber was measured using radiochromic films and alanine dosimeters behind the lead shielding. The radiation absorbed by the fibers in front of the shielding was calculated from the known source strength and the distance between fiber and source. 
During the first period of 16.1 hours the radiation absorbed by each fiber was $380 \mathrm{Krad}$, and in the subsequent 22.5 hours $1.4 \mathrm{Mrad}$, for a total of about $1.8 \mathrm{Mrad}$. The exposure of the fibers was not uniform over their length: from geometry we estimate a non uniformity of about $25 \%$. Behind the lead wall, where the LED's , the lamp and the photodiodes and amplifiers were installed, the exposure to radiation was between 40 and $80 \mathrm{krad}$, measured at 6 different places.

The photodiodes also act as nuclear counters: photons from the Cobalt source get absorbed and cause a current. In the photodiodes which were equipped with DC amplifiers this effect was observed indeed. The effect could be used to monitor the radiation as well, something which we did not attempt however.

\section{CALIBRATION}

As mentioned in chapter 2 the LED's were monitored by a photodiode directly, without fiber in between. In that way we could measure changes in the system LED+photodiode+amplifier. The correction derived from the amplifier viewing directly the photodiodes is applied to all the other signals. Indeed the output signal from that amplifier was found to be not constant over time. There were three components in its response, which can be seen in figure 2 .

1. We observed a periodic fluctuation with a frequency of $1 / 24$ hours, the minimum occuring in the early morning. The amplitude of that fluctuation was $4 \%$. We interpret it as a possible temperature sensitivity.

2. The signal decreased by several percent over the observation time. That might be due to radiation damages in either LED or photodiode surface or in the electronics of the amplifier.

3. Comparing the different 24 hour periods we find a non periodic fluctuation of about $1 \%$. We attribute it to an instability within the amplifier.

We correct the response of all amplifiers for the periodic fluctuation and for the overall de-

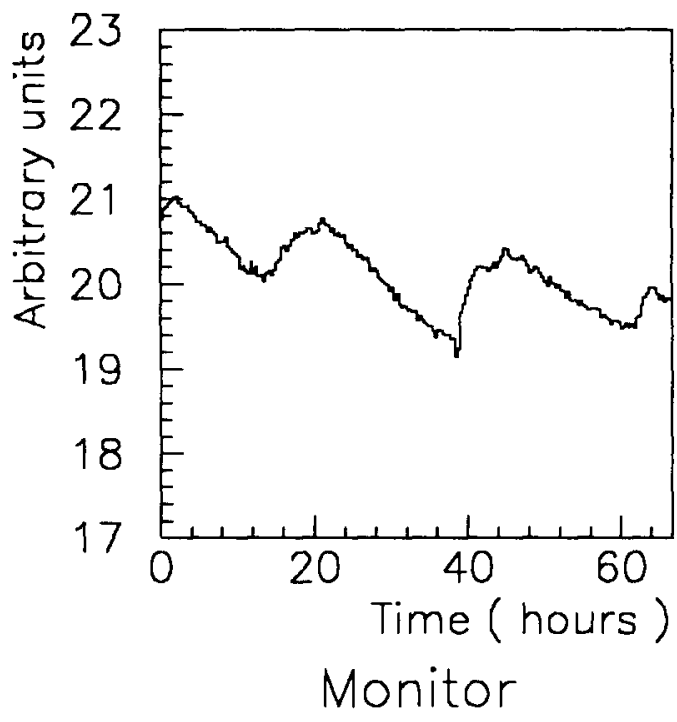

Figure 2: Output signal from the amplifier viewing directly the LED.

crease. The above mentioned non periodic fluctuation of $1 \%$ results in a systematic uncertainty of the corrected relative transmission of somewhat more than $1 \%$. We will quote it as $2 \%$ in the following as a reasonably conservative number.

For the time being we consider this precision more than sufficient. However, if needed, it could be increased feeding test-pulses to all amplifiers instead of one only.

\section{MEASUREMENT AND RESULTS}

We had 4 different types of fibers installed. Bicron 12 (scintillating fiber), Bicron 91A (wavelength shifter), Bicron 99-39 (wavelength shifter) and Kyowa SCF81 (scintillating). Figure 3 shows the change in transmission with time of the three Bicron fibers for green light.

The vertical axis of figure 3 is the relative transmission, normalized to 1 in the first bin. It is pedestal subtracted and corrected for instabilities. Figures 4 and 5 show the transmission of the Kyowa SCF81 and Bicron 99-93 fibers. We 


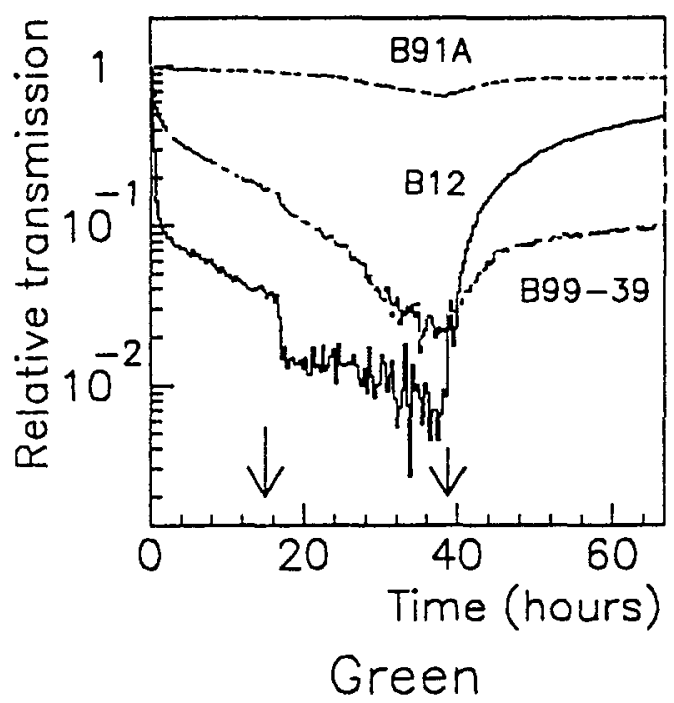

Figure 3: Change in transmission for green light. The arrows indicate the time when the intensity of the source was increased, and when the source was switched off.

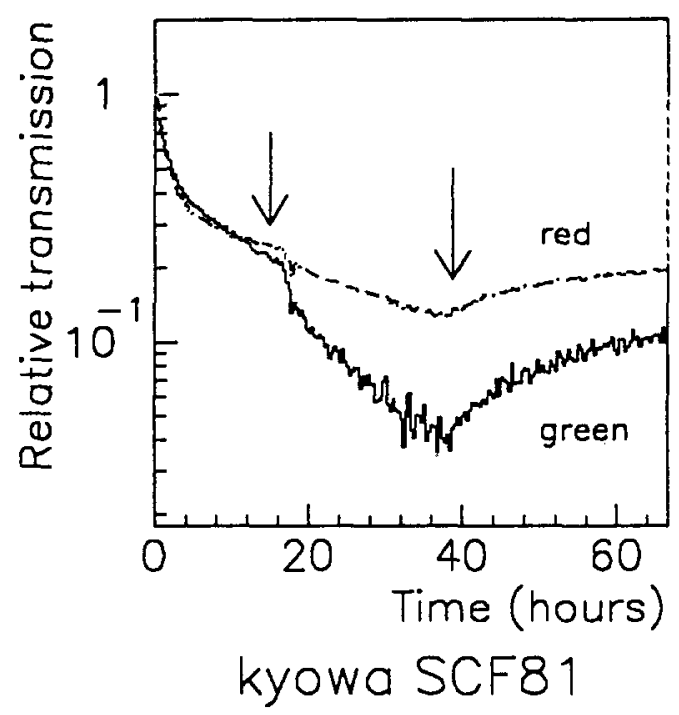

Figure 4: Change in transmission for green and red light, Kyowa SCF81 fiber.

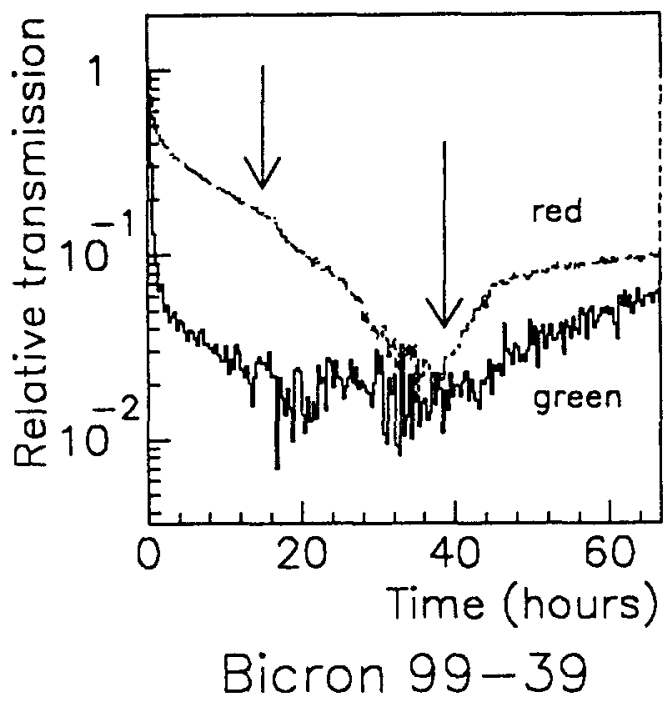

Figure 5: Change in transmission for green and red light, Bicron 99-39 fiber.

observe a fast decrease in transmission immediately after the source was switched on, and we see the steepening of the slope $\Delta$ transmission/ $\Delta$ time when the intensity of the radiation was increased at $t=16.1$ hours. All the fibers show some recovery as soon as the source is switched off. The differences in radiation damage for different fibers are considerable. Also the radiation damage for green and red light is very different for different fibers. Another interesting effect is that $\Delta$ transmission $/ \Delta$ time reaches a maximum for all Bicron fibers at about $t=25$ hours. The effect is not very strong, but it seems to confirm a similar observation which was made during the first engineering run described in ref.1.

To demonstrate the precision which we can obtain with our setup we show on a more expanded time scale the transmission of the fiber which was connected to the continuous light source around $t=39$ hours. The fiber used is Bicron 99-39. At that time we had the source switched off and shortly afterwards reactivated for a short time, as described in chapter 5 . We see the prompt response of the fiber to the radi- 


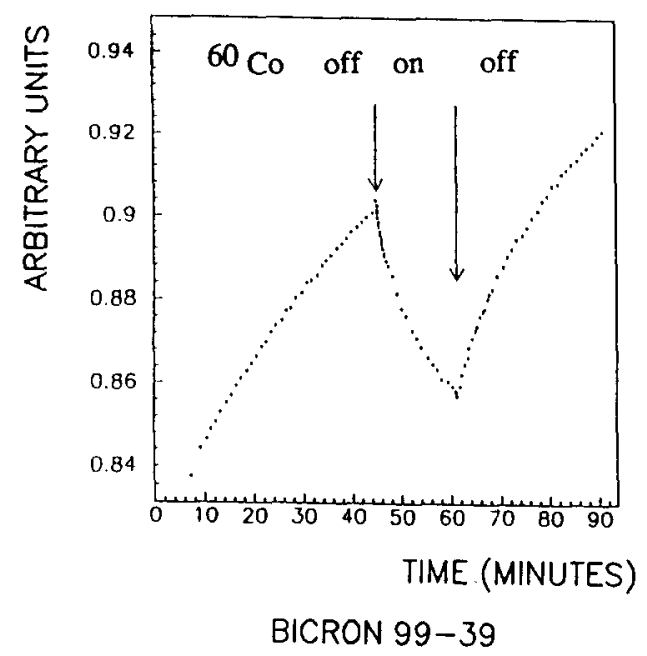

Figure 6: Fiber response on expanded time scale, Bicron 99-39 fiber.

ation on the smaller time scale in figure 6 with a precision of several permille. At the times when the source was switched on or off there is a small but significant discontinuity. From a comparison with a photodiode which was not connected to any fiber we know that this discontinuity is due to the photocurrent produced by the photons of the cobalt source. The photodiode was read out by a DC amplifier.

From figure 6 we see that both the radiation damage and the recovery proceed very fast in the first minutes of irradiation or recovery.

\section{CONCLUSION}

We observed the transmission of a number of optical scintillating and wavelenght shifting fibers over a time of 39 hours, integrating 1.8 Mrad of radiation from a ${ }^{\circ 0} \mathrm{Co}$ source. Their recovery was studied over 28 hours after irradiation. The measurement precision was about $2 \%$. We did the measurement for red and green light from LED's and for light emitted by an ordinary 4.5 Volt lamp. We saw very large difference in the highly non-linear radiation damage of the different fibers. We know ( see ref.3 ) that the basic material for all the three Bicron fibers is the same (Polystyrene): only their dopant is different. From figure 3 we can argue that it is the dopant which contributes mostly to the radiation damage. Also the development of the radiation damage for green and red light is very different for different fibers.

In some cases large recovery effects are observed on a time scale of several minutes only. The peculiar structure in $\Delta$ transmission/ $\Delta$ time which is already described in ref. 1 again is visible.

The setup is designed in a way which would allow its upgrading so that a large number of fibers could be measured simultaneously. The various components of the system are continuously calibrated and therefore one can potentially extend the measurement over a very long period of time without compromising the messurement precision.

\section{ACKNOWLEDGMENT}

We want to thank M.Atac (Fermilab) for many profitable discussions. J.Ernwein (Saclay) provided us with dosimeters and much helpful advice. The support of V.Cavasinni (Univ.Pisa) and G.Bellettini (Univ.Pisa) was crucial for a speedy realisation of the experiment. Special thanks go to M.Lora (INFN, CNR Legnaro) who is responsible for the ${ }^{60} \mathrm{Co}$ source, for his friendly support. C.Hurlbut and F. Kniest from Bicron gave us some of their newly developed fibers.

\section{REFERENCES}

1. "Measurement of Radiation Damage during Irradiation" in Proceedings of the Second international workshop on calorimetry in high energy physics, Capri, September 1991. Presentation by H.Grassmann, to be published.

2. "Instruction manual for Cobalt 60 irradiation unit", Atomic Energy of Canada Limited.

3. F.Kniest, Bicron, private communication 